\title{
Impacto de la luz artificial sobre la anidación de la tortuga marina Dermochelys coriacea (Testudines: Dermochelyidae), en playa Cipara, Venezuela
}

\author{
María Rondón Médicci ${ }^{1}$, Joaquín Buitrago ${ }^{2} \&$ Michael Mccoy ${ }^{1}$ \\ 1. Instituto Internacional en Manejo y Conservación de Vida Silvestre, Universidad Nacional de Costa Rica, Heredia- \\ Costa Rica; m_medicci@yahoo.com; mmccoycolton@yahoo.com \\ 2. EDIMAR-FLASA, Punta de Piedras, Margarita, Venezuela; jbuitrago@edimar.org
}

\author{
Recibido 07-VII-2008. Corregido 22-II-2009. Aceptado 25-III-2009.
}

\begin{abstract}
Impact of artificial light on nesting in the leatherback turtle Dermochelys coriacea (Testudines: Dermochelyidae) at Cipara beach, Venezuela. The number of Leatherback turtle nests and their spatial distribution was compared between years with and without artificial light, and between dark and lighted beach segments, in Cipara Beach, Paria Peninsula, Venezuela. Residents were interviewed to identify their perceptions about the impact of artificial light on sea turtles. Mean volume of sand per meter of beach was larger at La Peña, Cipara and La Remate and smaller at Varadero $(\mathrm{p}<0.001)$, increasing from April to June and later decreasing until August $(\mathrm{p}<0.05)$. Mean percentage of gravel was higher at Varadero and La Peña, and lower at La Remate and Cipara. Most interviewed people said that artificial light does not affect sea turtles. Between 2000 and 2005, 1217 leatherback landings and 1056 nests were observed. Successful nests increased with the years $(\mathrm{p}=0.035)$ as well as total nest number ( $\mathrm{p}=0.015$ ). From 2000 through 2003 there were 743 landings, 661 nests and 374 clutches. During the two years with electric light (2004-2005), there were 474 landings, 395 nests and 232 clutches. Proportion of landings with nest building decreased significantly during the years with electric light $(\mathrm{p}=0.005)$, but nesting success did not vary $(\mathrm{p}=0.402)$. No significant difference was found between landings per beach meter in dark and lighted sectors $(p=0.244)$, between nests built $(p=0.379)$ and in the rate of successful nesting $(\mathrm{p}=0.516)$. Dark and lighted sectors did not differ in the proportion of landings with nest building $(\mathrm{p}=0.067)$ and success rate $(\mathrm{p}=0.833)$. Rev. Biol. Trop. 57 (3): 515-528. Epub 2009 September 30.
\end{abstract}

Key words: Dermochelys coriacea, Cipara Beach, nesting, artificial lighting, leatherback, Venezuela, sea turtles.

En la actualidad, de las siete especies de tortugas marinas, seis están catalogadas como "En Peligro Crítico", "En Peligro" o "Vulnerables" en la Lista Roja de la IUCN (GETM-CSE/UICN 1995, Flemming 2001, IUCN 2008). Dentro de las principales causas que influyen en la disminución de las poblaciones de tortugas marinas están la captura tanto directa como incidental de adultos por parte de la pesquería (Guada \& Solé 2000, Spotila et al. 2000, Hernández 2002, Kennett et al. 2004), alteración de hábitats de alimentación y recolección ilegal de huevos (Spotila et al.
2000). De la misma manera, influye la alteración y pérdida de playas de anidación (Kennett et al. 2004), por desarrollo costero (Spotila et al. 2000, Flemming 2001, Deem et al. 2007), extracción de arena en las playas de anidación (Guada \& Solé 2000, Hernández 2002) y efectos de la iluminación artificial en la playa (Witherington 2000, Francia 2004, Deem et al. 2007).

La alteración del hábitat de anidación ha ido incrementando notablemente (Witherington 2000). Las playas han sufrido la presión de una creciente demanda (Chacón \& Arauz 2001) 
para el desarrollo de actividades turísticas, construcción de hoteles, restaurantes y residencias (Lutcavage et al. 1997, Parra 2002), implicando cambios en la estructura física de las playas y un incremento de la luz artificial (Chacón \& Arauz 2001).

Se ha documentado que la iluminación artificial en las playas, afecta la anidación de las tortugas marinas y produce la desorientación de los neonatos emergentes, quienes desvían su camino al mar por seguir las señales lumínicas (Raymond 1984, Witherington 2000, Silman et al. 2002, Francia 2004, Bourgeois 2007, Deem et al. 2007). Raymond (1984), reportó una drástica reducción en los intentos de anidación por las tortugas caguamas (Caretta caretta, Linnaeus 1758), en una playa de Florida, con luces muy brillantes. Mortimer (1982), describió como las tortugas verdes (Chelonia mydas, Linnaeus 1758), en Isla Ascensión, evitaban playas con luces artificiales. Salmon et al. (1995) encontraron que las tortugas caguamas, que anidan en playas donde el brillo de la luz urbana es visible detrás de las dunas, tienden a preferir áreas oscuras donde las construcciones se perfilan en contra del brillo artificial.

En Playa Junquillal, Pacífico de Costa Rica, el mayor número de anidaciones estuvo frente a construcciones oscuras, seguida por la playa oscura (50\% menos) y la playa iluminada (93\% menos). De igual manera, la proporción de deserciones fue más alta en las secciones de playa iluminadas (Francia 2004). En la costa este central de Florida a lo largo de $40 \mathrm{~km}$ de playa y durante 11 años, las anidaciones de caguama y tortuga verde (pero no de cardón, Dermochelys coriacea, Vandelli 1761), fueron menores en las áreas con más desarrollo humano (Weishampel et al. 2003). En el Parque Nacional Santa Rosa, Costa Rica, la tortuga negra (Chelonia mydas agassizii) fue indicada como "altamente sensible a personas en movimiento en la playa y a luces" (Cornelius 1986). Durante la temporada de anidación 2006-2007, en el Parque Nacional Pongara, Gabón, Deem et al. (2007) encontraron, a lo largo de $5 \mathrm{~km}$ de playa, 71 hembras de tortuga cardón desorientadas por la luz artificial.
Recientemente, en la localidad de Cipara, Península de Paria, costa noreste de Venezuela, se instaló la luz eléctrica. Por otra parte, la franja de vegetación que está frente a la playa ha sufrido durante los últimos años una intensa tala para la construcción de ranchos en la playa. Esto causa cambios en la barrera natural que bloquea, en algunos sectores, la incidencia directa de la luz artificial sobre la playa. Como se ha visto en otras partes del mundo, la iluminación en la playa de Cipara puede traer varias consecuencias. Esto incluye la disminución en el número de hembras anidando o el número de anidaciones exitosas. Además, puede provocar un cambio en el comportamiento de anidación de las tortugas marinas, propiciando la anidación en los sectores con menor incidencia de luz artificial, pero donde otras características que afectan el éxito reproductivo pueden ser peores. O simplemente desplazando la anidación hacia otras playas cercanas.

En el presente estudio se hizo una evaluación del impacto de la luz artificial sobre la anidación de la tortuga cardón en la playa de Cipara, Venezuela. Los objetivos fueron estimar el número de salidas (con nidos $\mathrm{y}$ sin nidos) y su distribución espacial a lo largo de la playa en los años sin iluminación (20002003) y con iluminación artificial (2004-2005) y en los segmentos de playa iluminados y los oscuros en los años con iluminación artificial (2004-2005).

\section{MATERIALES Y MÉTODOS}

La localidad de Cipara $\left(62^{\circ} 42^{\prime} \mathrm{W}, 10^{\circ} 45^{\prime} \mathrm{N}\right)$, se ubica en la parte noreste de la Península de Paria, Estado Sucre, Venezuela (Guada et al. 2002). La playa mide $2060 \mathrm{~m}$ de largo y comprende cuatro sectores, conocidos localmente como Varadero (220 m), Cipara (740 m), La Peña (600 m) y La Remate (500 m) (Fig. 1).

Los cuatro sectores presentan diferentes características que los discriminan, tanto ambientalmente como con respecto a la variable independiente estudiada: la incidencia de la iluminación artificial. Varadero y La Peña presentan arena gruesa y pendientes pronunciadas 


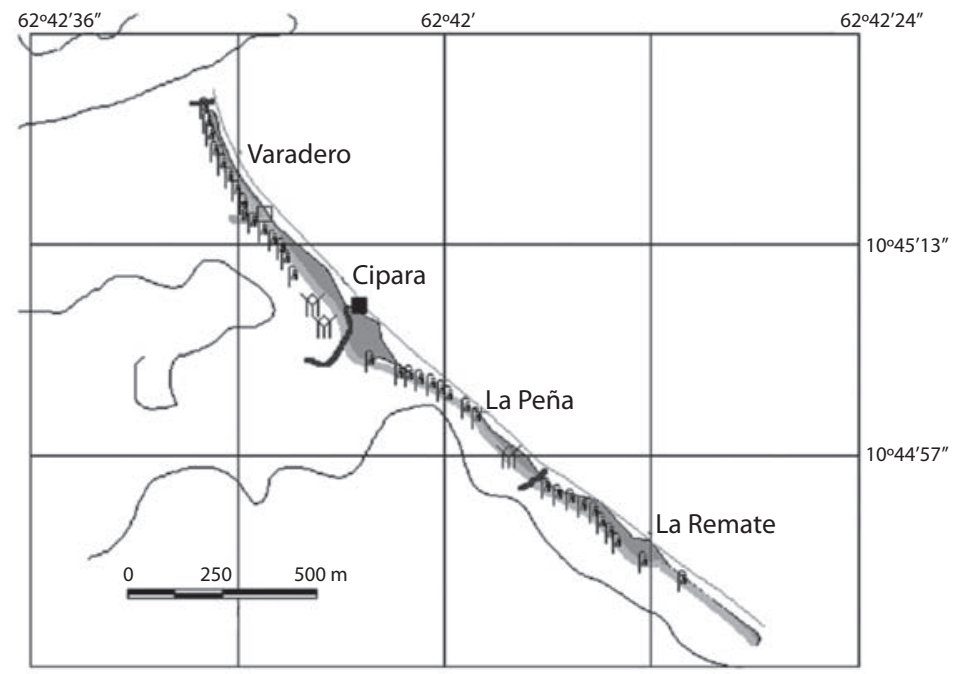

Fig. 1. Esquema de Playa Cipara mostrando, los sectores de la playa, los postes de alumbrado público, la vialidad (gris claro) y la vegetación costera (gris oscuro), el cuadrado negro muestra la ubicación del vivero y el cuadro claro un cobertizo costero.

Fig. 1. Cipara beach scheme showing beach sectors, public ligth poles, roads (ligth gray) and coastal vegetation (dark gray), black square shows hatchery location and white square a coastal hut.

entre junio y septiembre (Guada et al. 2002) y en ellas se encuentran la mayoría de las viviendas. La vegetación frente a la playa en estos dos sectores, principalmente uva de playa (Coccoloba uvifera), ha sufrido una intensa tala, abriendo espacio que permite incidencia directa de luz artificial sobre la playa. En La Peña existe un arrecife rocoso, conocido localmente como "el muelle", de aproximadamente $300 \mathrm{~m}$ de largo que puede bloquear la llegada a la playa de las cardón. Los sectores Cipara y La Remate tienen arena fina y pendientes suaves durante todo el año (Guada et al. 2002). La vegetación frente a la playa está representada principalmente por uva de playa y la tala en estos sectores no ha sido tan intensa como en los otros dos. Durante la época de lluvia (junionoviembre) irrumpen dos ríos, El Tigre y Cipara, en La Peña y Cipara, respectivamente.

La comunidad de Cipara cuenta con 48 viviendas habitadas. En junio del 2003 se instaló la luz eléctrica. El alumbrado público fue colocado, con una distancia de $40 \mathrm{~m}$ entre postes, a lo largo del camino que recorre toda la extensión de la playa.

Se midió el perfil de la playa estimando mensualmente el volumen de arena (Emery 1961), para cada sector (Varadero, Cipara, La Peña y La Remate), desde abril hasta agosto del 2005. Se hizo el levantamiento de la línea de marea media, la faja de vegetación y el camino, utilizando un receptor del Sistema de Posicionamiento Global (GPS). Se caracterizó el tamaño del grano de sedimento por granulometría por tamizado en columna Tyler (Llano 1987, Hernández 2000), por sector y a tres niveles: zona baja, media y alta de la playa.

La playa se sectorizó en 14 segmentos de $150 \mathrm{~m}$ cada uno con fines de posicionar las salidas. De abril a agosto de cada año entre 2000 y 2005, se efectuaron recorridos nocturnos a lo largo de la playa, desde las 21:00 hasta las 5:00 hr. Para cada tortuga vista o rastro dejado por la tortuga (cuando esta no se pudo observar), se registró: a) el tipo de salida: 1) nido con huevos o nidada, 2) nido sin huevos, 3 ) nido 
sin identificar (movimiento de arena pero no se sabe si hubo desove o no) y 4) salidas sin nidos (Schroeder y Murphy 2000) y, b) la distribución espacial del tipo de salida según el sector y el segmento de playa en que ocurrió.

Para evaluar los efectos de la iluminación artificial en las actividades de anidación se definieron dos tratamientos a lo largo de la playa: 1) cuatro segmentos de playa oscuros (1 $013 \mathrm{~m}$ en total), donde por condiciones de vegetación y/o topografía no llega la luz artificial a la playa y 2) cuatro segmentos de playa iluminados (1 $047 \mathrm{~m}$ en total), con fuente de luz artificial permanente durante toda la noche (Fig. 2). Con el fin de evitar el efecto de borde no se usaron los sectores de $150 \mathrm{~m}$ de largo como unidades, sino sólo aquellas porciones de ellos claramente fuera de la influencia lumínica del sector vecino, por lo que quedaron 892.5 $\mathrm{m}$ clasificados como oscuros y 307.5 como iluminados.

Entre el 21 de diciembre del 2005 y el 03 de enero del 2006, se realizó una evaluación acerca de la percepción de los residentes costeros, sobre la influencia de la luz artificial en la anidación de las tortugas marinas, utilizando la encuesta como método cuantitativo de la investigación social (Cea 1998), en la localidad de Cipara y otras localidades adyacentes (Unare, San Juan de las Galdonas y Querepare) donde también existe iluminación artificial que incide en la playa. El cuestionario se dividió en dos preguntas: 1) ¿Considera que la luz eléctrica que alumbra las playas afecta a las tortugas marinas? y 2) ¿Por qué?

Para los análisis estadísticos de los datos de anidación por año, sector e iluminación se utilizó el programa Statgraphics Plus 5.1 ${ }^{\mathrm{TM}}$. Las respuestas al cuestionario fueron analizadas con el programa SPSS 14.0 ${ }^{\mathrm{TM}}$. En todos los casos el nivel de significación empleado $\alpha$ fue 0.05 .

\section{RESULTADOS}

Durante las temporadas 2000-2005 se registraron un total de 1217 salidas de tortuga cardón; 1056 con nidos y 161 sin nidos. Del total de salidas, 17 fueron diurnas; diez con huevos y siete sin. El número de nidos con huevos y nidos sin huevos dependió significativamente del año de anidación $\left(\mathrm{X}_{\mathrm{GL}}^{2}=11.96\right.$, $\mathrm{p}=0.035)$. El año 2000 tuvo el menor número de nidos sin huevos. El número de nidos totales y salidas sin nidos dependieron igualmente de los años de anidación $\left(\mathrm{X}_{\mathrm{GL5}}^{2}=14.12, \mathrm{p}=0.015\right)$. Las salidas, tanto con nidos como sin nidos aumentaron año tras año, durante el periodo de estudio (Cuadro 1).

En los años previos a la instalación de electricidad (2000-2003) se observaron 743 salidas, de las cuales 661 produjeron anidación y 374 nidada confirmada. En los dos años con iluminación artificial estudiados (2004-2005), se contaron 474 salidas con 395 nidos y 232 nidadas (Cuadro 1). La proporción de salidas

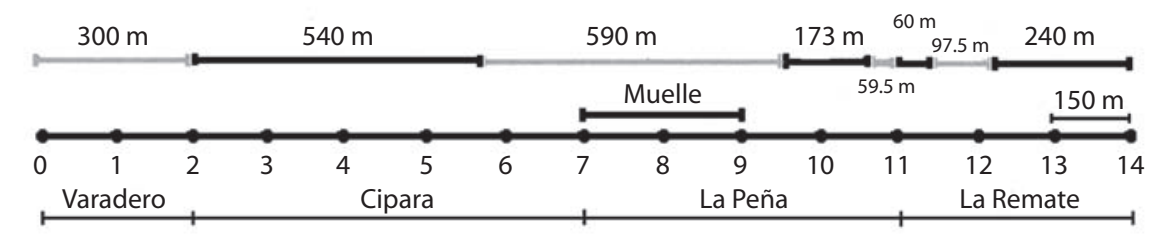

Con luz $1047 \mathrm{~m}$

Sin luz $1013 \mathrm{~m}$

Fig. 2. Diagrama de los tratamientos empleados para evaluar el efecto de la iluminación artificial en la anidación de la tortuga cardón (D. coriacea), en Playa Cipara, Península de Paria, Venezuela.

Fig. 2. Diagram of treatments emploied to evaluate artificial light effects in Leatherback (D. coriacea) nesting at Cipara beach, Venezuela. 
CUADRO 1

Número de salidas con nidos (con huevos, sin huevos, sin identificar y totales) y sin nidos y su porcentaje de las salidas por año, de la tortuga cardón (D. coriacea), durante los periodos de anidación 2000-2005, en Playa Cipara, Península de Paria, Venezuela

TABLE 1

Number of emergences with nests with and without eggs, without data, total nest number and with out nests and the percentage of yearly emergences of leatherback turtles (D. coriacea), between the years 2000-2005 at Cipara beach, Paria Peninsula, Venezuela

\begin{tabular}{ccccccc} 
Año & $\begin{array}{c}\text { Nidos con } \\
\text { Huevos }(\%)\end{array}$ & $\begin{array}{c}\text { Nidos sin } \\
\text { Huevos }(\%)\end{array}$ & $\begin{array}{c}\text { Sin } \\
\text { Identificar }(\%)\end{array}$ & $\begin{array}{c}\text { Total } \\
\text { nidos }\end{array}$ & $\begin{array}{c}\text { Sin Nidos } \\
(\%)\end{array}$ & $\begin{array}{c}\text { Total } \\
\text { Salidas }\end{array}$ \\
\hline 2000 & $61(55)$ & $14(13)$ & $27(25)$ & 102 & $8(7)$ & 110 \\
2001 & $108(52)$ & $60(29)$ & $22(11)$ & 190 & $16(8)$ & 206 \\
2002 & $104(52)$ & $57(28)$ & $15(7)$ & 176 & $25(12)$ & 201 \\
2003 & $101(45)$ & $64(28)$ & $28(12)$ & 193 & $33(15)$ & 226 \\
2004 & $119(46)$ & $62(24)$ & $35(14)$ & 216 & $43(17)$ & 259 \\
2005 & $113(53)$ & $45(21)$ & $21(10)$ & 179 & $36(17)$ & 215 \\
Total & $606(0.50)$ & $302(25)$ & $148(12)$ & 1056 & $161(13)$ & 1217
\end{tabular}

con construcción de nido disminuyó significativamente (Proporción binomial, $Z=2.783$, $\mathrm{p}=0.005$ ) en los años con luz eléctrica, pero no varió el éxito de desove (proporción de nidos construidos, con desove efectivo) (Proporción binomial, $\mathrm{Z}=-0.838, \mathrm{p}=0.402$ ).

En playa Cipara la distribución de las nidadas no fue homogénea a lo largo de la playa (Fig. 3). El número de nidos y salidas sin nidos, en los diferentes años, fue mayor en el sector de Cipara. En el sector de Varadero, a excepción del 2002, no hubo nidos con desove. En Cipara el número de nidos sin huevos fue aumentando hasta el 2002, disminuyendo posteriormente hasta el 2005. En el 2005, el número de nidos sin desove en La Remate fue mayor que en $\mathrm{La}$ Peña. En Varadero, en el 2002 el número de nidos sin huevos fue mayor que en La Remate y La Peña (Fig. 3).

Los ANOVA, previa corroboración de la homogeneidad de las varianzas, para el número de salidas totales por metro de playa, dieron significativamente diferente entre Varadero y la Peña, con La Remate y Cipara (prueba de ámbitos múltiples Tuckey $\mathrm{HSD}),\left(\mathrm{F}_{(3,16)}=\right.$ $103.11, \mathrm{p}<0.0001)$. Lo mismo ocurre para la densidad de salidas sin construcción de nido $\left(\mathrm{F}_{(3,16)}=6.86, \mathrm{p}=0.0035\right)$ y para las anidaciones exitosas por metro de playa $\left(\mathrm{F}_{(3,16)}=82.26\right.$, $\mathrm{p}<0.0001)$. Para la densidad de nidos construidos sin desove, la diferencia es significativa entre el sector Cipara y los otros tres sectores $\left(\mathrm{F}_{(\mathrm{GL} \mathrm{3,16)}}=8.29, \mathrm{p}=0.0015\right)$ (Fig. 4).

La proporción de anidaciones exitosas fue igual entre los 4 sectores (Proporciones Binomiales, $\left.\mathrm{X}_{\mathrm{GL} 3}^{2}=4.97, \mathrm{p}=0.174\right)$, al igual que la proporción de salidas que terminaron en la construcción de nidos $\left(\mathrm{X}_{\mathrm{GL} 3}^{2}=2.82, \mathrm{p}=0.42\right)$ y la proporción de salidas sin construcción de nido $\left(\mathrm{X}_{\mathrm{GL} 3}^{2}=0.52 \mathrm{p}=0.915\right)$. Si se encontró diferencia significativa entre los sectores en la proporción de anidaciones no exitosas, es decir con construcción de nido pero sin desove $\left(\mathrm{X}_{\mathrm{GL} 3}^{2}=16.85, \mathrm{p}=0.0008\right)$.

En los años con iluminación artificial (2004-2005), en los segmentos oscuros el $78 \%$ de los nidos fueron con huevos y el $22 \%$ sin huevos, mientras en los mismos años, en los segmentos iluminados la mitad fueron nidos con huevos y la otra mitad nidos sin huevos (Cuadro 2).

No se encontró diferencia significativa entre el número total de salidas por metro de playa en los sectores oscuros y los iluminados para los años 2004 y $2005\left(\mathrm{~F}_{(1,9)}=1.56, \mathrm{p}=0.244\right)$, ni entre el número de nidos construidos $\left(\mathrm{F}_{(1,9)}=0.85\right.$, 

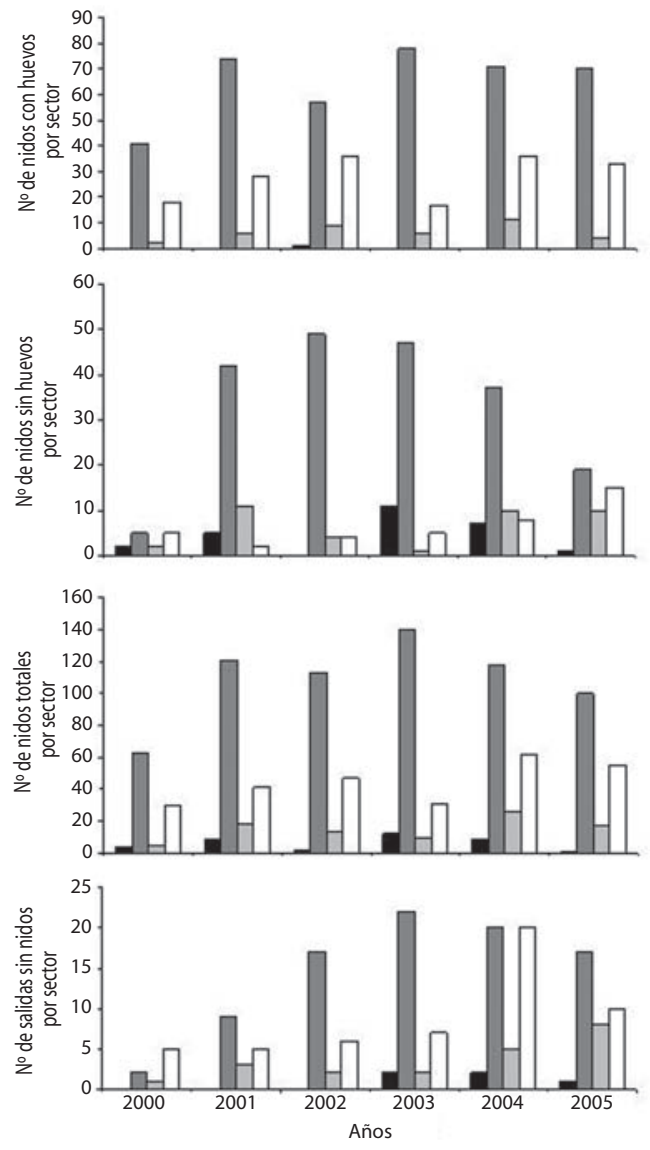

- Varadero a Cipara

- La Peña a La Remate

Fig. 3. Número de salidas con nidos (con huevos, sin huevos y totales) y sin nidos de la tortuga cardón $(D$. coriacea), por sector por año (2000-2005), en la playa de Cipara, Venezuela.

Fig. 3. Number of nests with and without eggs, total nest and non-nesting emergences the leatherback turtle $(D$. coriacea), by sector and year (2000-2005), in Cipara beach, Venezuela.

$\mathrm{p}=0.379)$, ni entre las anidaciones con desove $\left(\mathrm{F}_{(1,9)}=0.46, \mathrm{p}=0.516\right)$ (Cuadro 3).

De la misma manera no se encontró diferencia significativa en la proporción de las salidas totales que terminaron en anidación (Proporciones Binomiales, $\mathrm{Z}=1.83$, $\mathrm{p}=0.067$ ) entre los sectores iluminados y oscuros, ni en la proporción de nidos exitosos (con
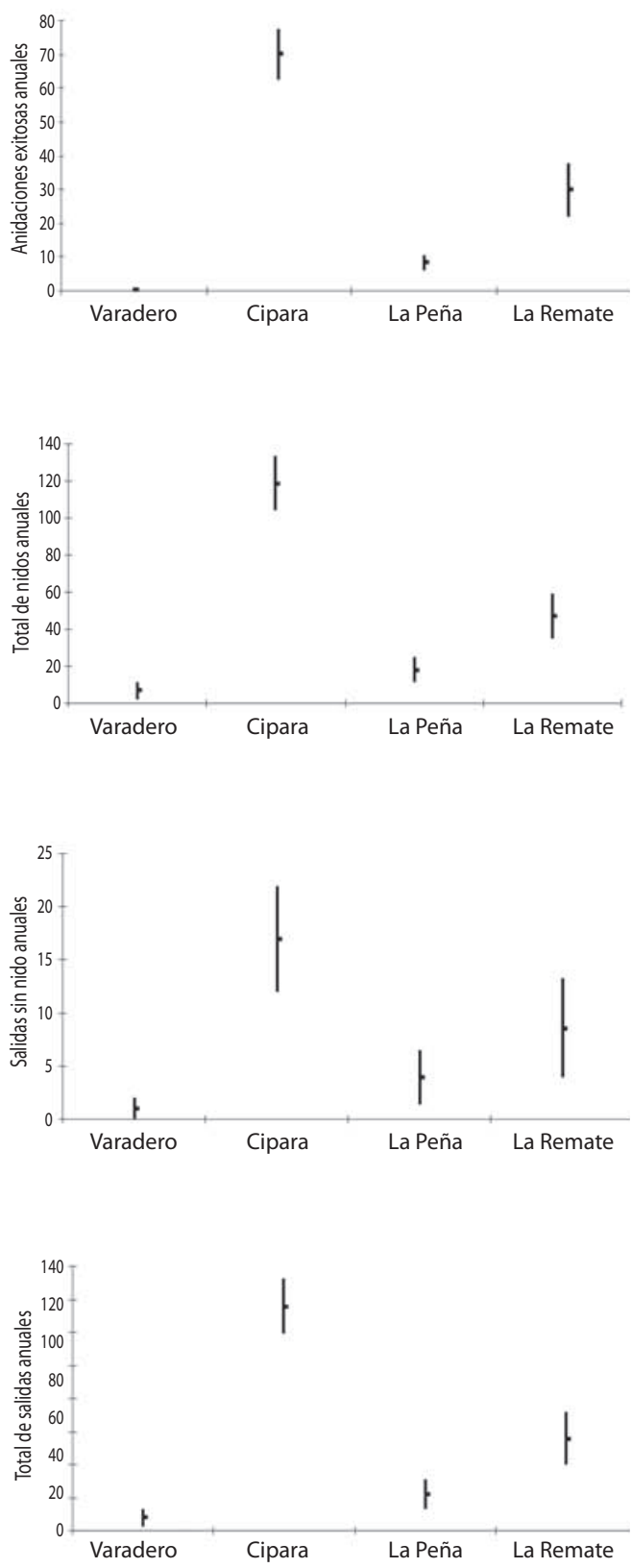

Fig. 4. Número de salidas con nidos (con huevos, sin huevos y totales) y sin nidos de la tortuga cardón (D. coriacea) (media anual e intervalos de $\pm 1 \mathrm{DE}$ ), en los diferentes sectores de la playa de Cipara, Venezuela.

Fig. 4. Number of nests with and without eggs, total nest and non-nesting emergences of leatherback turtle ( $D$. coriacea) (annual mean and bars of $\pm 1 \mathrm{SD}$ ), at the different sectors, of Cipara beach, Venezuela. 


\section{CUADRO 2}

Número y porcentaje de salidas con nidos (con huevos, sin huevos, sin identificar y totales) y sin nidos de la tortuga cardón (D. coriacea) en los segmentos de playa oscuros e iluminados, durante los períodos de anidación 2004-2005, en Playa Cipara, Península de Paria, Venezuela

TABLE 2

Number and percentage of nests with and without eggs, without data, total nest number and non-nesting emergences of leatherback turtles (D. coriacea), in dark and lighted beach segments, during years 2004-2005 at Cipara beach, Paria Peninsula, Venezuela

$\begin{array}{lccccc}\text { Segmentos } & \begin{array}{c}\text { Nidos con } \\ \text { Huevos }(\%)\end{array} & \begin{array}{c}\text { Nidos sin } \\ \text { Huevos }(\%)\end{array} & \begin{array}{c}\text { Sin } \\ \text { Identificar (\%) }\end{array} & \text { Total } & \begin{array}{c}\text { Sin } \\ \text { Nidos }(\%)\end{array} \\ \text { Iluminados } & 30(19) & 31(46) & 6(26) & 67 & 13(33) \\ \text { Oscuros } & 129(81) & 36(54) & 17(74) & 182 & 26(67) \\ \text { Total } & 159(100) & 67(100) & 23(100) & 249 & 39(100)\end{array}$

desove) (Proporciones Binomiales, $\mathrm{Z}=0.211$, $\mathrm{p}=0.833)$.

Un análisis de componentes principales de los resultados de los diferentes tipos de salidas absorbe el $98.2 \%$ de la variabilidad en los tres primeros componentes y el $85.4 \%$ en los dos primeros ejes (Fig. 5). El componente 1, que participa con $61.3 \%$ de la variabilidad está claramente definido hacia valores negativos por las anidaciones no exitosas, las cuales están vinculadas a las zonas iluminadas (Cipara 5, Cipara 6, La remate 11, La remate 12) aunque algunas zonas no iluminadas de La Remate presentan resultados similares.

La selección del sitio de anidación, y una vez construido el nido, si desova o no, es sin

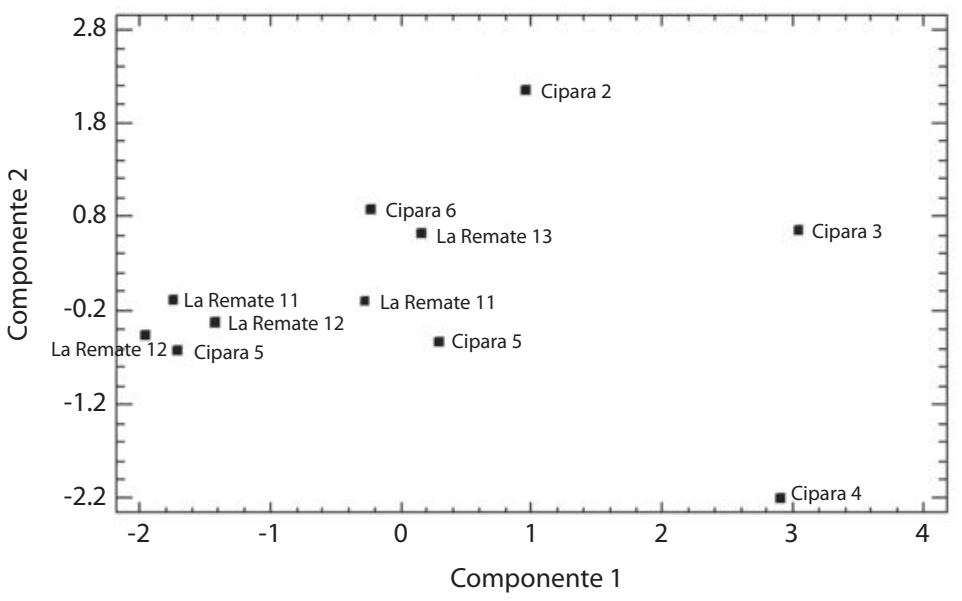

Fig. 5. Diagrama de dispersión de los sectores de playa estudiados en el plano de los dos primeros componentes principales formados por los resultados de los diferentes tipos de salidas en sectores con iluminación artificial (mayúsculas) y sin iluminación artificial en Cipara, Paria, Venezuela. Los dos ejes absorben el $85 \%$ de la variabilidad. El eje uno está dominado hacia valores negativos por la cantidad de anidaciones no exitosas.

Fig. 5. Scatterplot of beach sectors in the first two principal components formed by female activities in artificially lighted (capital font) and dark sectors in Cipara, Paria, Venezuela. The first two axis account for $85 \%$ of the variability. The first axis is dominated towards negative values by the amount of nests without a clutch. 
CUADRO 3

Densidad de anidación ( ${ }^{\circ}$ de salidas/m de playa) y porcentaje de nidos (con huevos, sin huevos, sin identificar y totales) y salidas sin nidos de la tortuga cardón (D. coriacea), en los segmentos de playa oscuros e iluminados, en los sectores Cipara y La Remate, durante los periodos de anidación 2004-2005, en Playa Cipara, Península de Paria, Venezuela

TABLE 3

Nesting density ( $n^{\circ}$ of landings/m of beach) and percentage of nests with and without eggs, total nest and non-nesting emergences of leatherback turtles (D. coriacea), in dark and lighted segments at Cipara and La Remate, between the years 2000-2005 in Cipara beach, Paria Peninsula, Venezuela

$\begin{array}{lccccc}\text { Segmentos } & \begin{array}{c}\text { Con } \\ \text { Huevos }(\%)\end{array} & \begin{array}{c}\text { Sin } \\ \text { Huevos }(\%)\end{array} & \begin{array}{c}\text { Sin } \\ \text { Identificar (\%) }\end{array} & \text { Total } & \begin{array}{c}\text { Sin } \\ \text { Nidos (\%) }\end{array} \\ \text { Iluminados } & 0.37(30) & 0.16(37) & 0.02(7) & 0.55 & 0.01(6) \\ \text { Oscuros } & 0.88(70) & 0.27(63) & 0.27(93) & 1.28 & 0.16(94) \\ \text { Total } & 1.25(100) & 0.43(100) & 0.29(100) & 1.83 & 0.17(100)\end{array}$

duda una decisión multivariable. En un intento por definir cuales de las variables independientes controladas influían en la anidación y su éxito, se corrió una regresión múltiple con cada uno de los diferentes tipos de salida como variable dependiente y el sector, la condición de iluminación, el porcentaje de arena y la extensión de cada segmento de playa, como variables independientes. Sólo se obtuvo una regresión significativa entre el número de nidos no exitosos (sin desove) y la condición de iluminación y la longitud del segmento $\left(\mathrm{R}^{2}=63.33, \mathrm{~F}_{(2,8)}=6.08, \mathrm{p}=0.025\right)$. La ecuación que representa esta relación es número de nidos sin desove $=-4.35883+2.12987^{*} \mathrm{Luz}+$ $0.074523 *$ Long.

Caracterización de la playa: El volumen medio de arena por metro cúbico de playa fue mayor en La Peña y Cipara y menor en La Remate y Varadero (ANOVA, $\mathrm{F}_{(3,19)}=12.41$, $\mathrm{p}=0.0001$ ) (Fig. $6 \mathrm{~B}$ ). El volumen medio de arena por metro cúbico de playa aumentó de abril a junio y posteriormente disminuyó un poco hasta agosto (ANOVA, $\mathrm{F}_{(4 \text { y 19) }}=4.43$, $\mathrm{p}=0.02$ ) (Fig. $6 \mathrm{~A})$.

En el sector La Remate se mantuvieron los volúmenes similares entre toda la temporada de anidación (ámbito 73.7-49.2 $\mathrm{m}^{3}$ ). Por el contrario, el sector La Peña tuvo un brusco aumento de $60-65 \mathrm{~m}^{3}$ en abril-mayo a $140-120$ $\mathrm{m}^{3}$ a partir de junio. En los otros sectores el aumento fue menor pero proporcional (aproximadamente el doble); en Cipara de 48-37 $\mathrm{m}^{3}$ en abril-mayo a 75-92 $\mathrm{m}^{3}$ a partir de junio. El sector Varadero por su parte también presentó un incremento de $28 \mathrm{~m}^{3}$ a $45 \mathrm{~m}^{3}$ en la misma temporada (Cuadro 4).

Varadero fue el sector con más pendiente durante toda la temporada, y Cipara el menos pendiente salvo en abril cuando La Remate tuvo menor declive. Todos los sectores, a excepción de La Remate, tuvieron el mismo comportamiento, la playa es más angosta y pendiente en abril que en los otros meses. En La Remate es muy similar todo el año.

El porcentaje medio de gravas fue mayor en Varadero y La Peña y menor en La Remate y en Cipara pero no significativamente (ANOVA, $\left.\mathrm{F}_{(3,8)}=1.96, \mathrm{p}=0.199\right)$. El porcentaje medio de gravas fue mayor en la zona infralitoral que en la zona de barrido y en la zona seca de la playa, pero la diferencia no es significativa (ANOVA, $\left.\mathrm{F}_{(2,9)}=1.71, \mathrm{p}=0.235\right)$ (Cuadro 5).

Percepción de los residentes costeros: Se entrevistaron a 246 personas, mayores de 18 años (73 en Cipara, 63 en Unare, 32 en San Juan y 78 en Querepare). En general, el $70.4 \%$ (133) de las personas entrevistadas consideraron que la luz artificial no afecta a las tortugas marinas. De ellos, $68 \%$ no supieron decir por qué la luz artificial no afecta a las tortugas marinas o no tienen ninguna razón en 

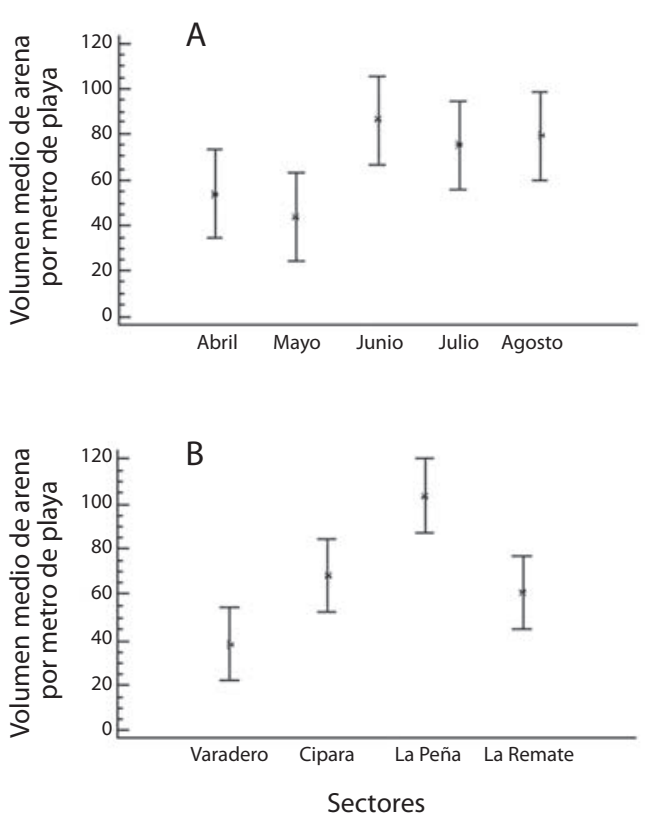

Fig. 6. Volumen medio de arena por metro cúbico de playa por mes (A) (abril-agosto) (con intervalos 95\% Tukey) y por sector (B) durante la temporada 2005, en la playa de Cipara, Península de Paria, Venezuela.

Fig. 6. Mean volume of sand per beach front meter by month (A) (April to August) and sector (B), during 2005, in Cipara beach, Paria Peninsula, Venezuela.

particular, $18.8 \%$ afirmaron que salen durante el día a poner sus huevos, $8.2 \%$ considera que la luz no les hace nada a las tortugas y $5.3 \%$ han observado que éstas siguen saliendo a la playa a poner huevos. El $28 \%$ (53) de personas entrevistadas consideraron que la luz artificial sí afecta a las tortugas marinas. De ellos, $43 \%$ afirmaron que se alejan de la playa y no salen a poner, $33.9 \%$ consideraron que las tortugas buscan la oscuridad para poner, $15 \%$ no supieron el por qué si afecta o no tuvieron ninguna razón de por qué la luz artificial si afecta a las tortugas marinas y el $7.5 \%$ consideraron que se desorientan y perjudican a los tortuguillas. Finalmente, el $1.6 \%$ de las personas entrevistadas no supieron si la luz artificial afecta o no a las tortugas marinas.

\section{DISCUSIÓN}

Un efecto atribuido a la iluminación artificial sobre la anidación de las tortugas es que éstas no salen del mar. Talbert et al. (1980), encontraron una disminución en las salidas en áreas de playa iluminadas, pero Mann (1977) encontró anidaciones producidas a pesar de edificaciones con iluminación. En Cipara se encontró que la diferencia entre los años con electricidad y sin ella, radica en la proporción de salidas con construcción de nido.

Se desconoce exactamente por qué la luz artificial afecta la anidación, Witherington \& Martin (2003) consideraron que las luces artificiales son interpretadas por las tortugas como luz diurna, lo cual afecta su comportamiento de anidación, que es usualmente nocturno. En Playa Cipara, y otras playas adyacentes,

\section{CUADRO 4}

Volumen de arena $\left(\mathrm{m}^{3}\right)$ por metro de frente de playa, de abril a agosto, en los sectores Varadero, Cipara, La Peña y La Remate, temporada 2005, en Playa Cipara, Península de Paria, Venezuela

TABLE 4

Volume of sand $\left(\mathrm{m}^{3}\right)$ per meter of beach front, between April and August, at Varadero, Cipara, La Peña and La Remate, during the year 2005, in Cipara beach, Paria Peninsula, Venezuela

$\begin{array}{lcccc}\text { Mes/Sector } & \text { Varadero } & \text { Cipara } & \text { La Peña } & \text { La Remate } \\ \text { Abril } & 28.00 & 47.98 & 65.60 & 73.70 \\ \text { Mayo } & 27.89 & 37.57 & 60.14 & 49.23 \\ \text { Junio } & 45.18 & 92.32 & 142.16 & 65.00 \\ \text { Julio } & 44.62 & 75.25 & 125.09 & 54.68 \\ \text { Agosto } & 45.16 & 87.18 & 123.64 & 62.00\end{array}$


CUADRO 5

Tamaño y clasificación de los granos de arena de la zona baja (1), media (2) y alta (3) en los sectores Varadero (V), Cipara (C), La Peña (LP) y La Remate (LR), temporada 2005, en Playa Cipara, Península de Paria, Venezuela

TABLE 5

Sand grain size classification in lower (1), middle (2) and upper beach zones (3) at Varadero (V), Cipara (C), La Peña (LP) and La Remate (LR), in the year 2005, in Cipara beach, Paria Peninsula, Venezuela

$\begin{array}{cccl}\text { Zona } & \text { \% Gravas } & \text { \% Arenas } & \text { Clasificación Shepard (1954) } \\ \text { V1 } & 100.00 & 0.00 & \text { GRAVAS } \\ \text { V2 } & 100.00 & 0.00 & \text { GRAVAS } \\ \text { V3 } & 45.63 & 54.37 & \text { ARENA GRAVOSA } \\ \text { C1 } & 67.43 & 32.57 & \text { GRAVAS } \\ \text { C2 } & 0.06 & 99.94 & \text { ARENAS } \\ \text { C3 } & 7.79 & 92.21 & \text { ARENAS } \\ \text { LP1 } & 76.24 & 23.76 & \text { GRAVAS } \\ \text { LP2 } & 53.84 & 46.16 & \text { GRAVA-ARENOSA } \\ \text { LP3 } & 99.91 & 0.09 & \text { GRAVAS } \\ \text { LR1 } & 89.47 & 10.53 & \text { GRAVAS } \\ \text { LR2 } & 14.75 & 85.25 & \text { ARENAS } \\ \text { LR3 } & 15.30 & 84.70 & \text { ARENAS }\end{array}$

las tortugas cardón pueden salir a anidar de día (Rondón 2003). Se ha observado que los cardones que salen en Cipara, durante el día, principalmente a horas cercanas del mediodía, raramente regresan a visitar esta playa. Quizás esto se deba que al salir de día, son perturbadas por los residentes (Williams-Walls et al. 1983, Murphy 1985, Witherington 1992) haciendo que se retiren a otras playas o que inversamente hayan sido perturbadas en otras zonas y hayan venido a esta playa a desovar. Worth \& Smith (1976) reportaron que las cabezonas perturbadas por personas y no pudieron anidar, salieron de nuevo en otras playas fuera de su área acostumbrada.

Existen otros factores de la iluminación que puede afectar a las tortugas marinas, como el tipo y la intensidad de luz. Witherington (1992), realizó experimentos en dos playas de anidación, Melbourne, Florida y Tortuguero, Costa Rica, con dos fuentes de luz diferentes (vapor de mercurio y vapor de sodio). Tanto las tortugas verdes como las cabezonas mostraron una tendencia significativa a evitar las áreas de playa alumbradas por luces de vapor de mercurio blancas. Sin embargo, no se detectó ningún efecto ocasionado por las luces amarillas de vapor de sodio. En Cipara, la luz de los postes de alumbrado público es incandescente, amarilla y de bombillos de baja intensidad (150 Watios).

En estudios de la sensibilidad espectral, de la orientación de las crías (Mrosovsky \& Carr 1967, Witherington \& Bjorndal 1991), las luces incandescentes de color amarillo, son consideradas de mínima a moderadamente nocivas para las tortugas marinas, pues emiten poca luz de onda corta (Witherington \& Martin 2003). Carr \& Ogren (1960), Mrosovsky \& Shettleworth (1975) \& Mann (1977) han encontrado que el estímulo visual de luces indirectas, afecta la orientación de los neonatos hacia el mar (Whiterington \& Martin 2003, Deem et al. 2007). En experimentos tanto en laboratorio como en el campo, los neonatos se desplazaron hacia luces artificiales brillantes (Mrosovsky \& Shettleworth 1975) y hacia los objetos brillantes en las playas (Carr 1962). 
En Cipara, se observó que al eclosionar uno de los nidos, en una sección del sector Cipara, donde incide más la luz, las crías se desorientaron y se dirigieron al camino y hacia las casas que están frente a la playa. Aunque el efecto de la iluminación incandescente amarilla puede ser mínimo, puede interferir con la orientación de las crías (Witherington \& Martin 2003), como es el caso de algunas secciones en Cipara.

Además de la luz artificial hay otros factores que pueden afectar la selección de sitios de anidación, como la presencia de obstáculos en la playa (Mortimer 1982), la pendiente (Wood \& Bjorndal 2000) y la granulometría de la arena (Mortimer 1990). En el sector La Peña existe un arrecife que cubre casi toda su extensión y tanto la granulometría como la pendiente varían fuertemente de un sector a otro.

Considerando que la calidad de la arena de la playa parece influenciar en el éxito reproductivo, se podría esperar que las tortugas usen la textura de la arena como un criterio en la selección del sitio de anidación (Mortimer 1990). Por otra parte, el diámetro medio los sedimentos influye en la disponibilidad y conductividad de los gases respiratorios, la temperatura y la humedad. Mortimer (1990) observó una relación significativa entre las características físicas y composición química del sedimento con respecto a la selección y la supervivencia de las nidadas de Chelonia mydas en diferentes playas de anidación.

Por otra parte, cuando la pendiente de una zona erosionada es muy abrupta, se reduce el acceso para la anidación (Witherington 2000), este es el caso de Varadero donde la pendiente es mayor que en los otros sectores, mientras que en Cipara la pendiente es menor, y en La Remate se mantuvo sin fuertes cambios durante toda la temporada. En el caso de La Peña el comportamiento especial, puede ser debido al afloramiento rocoso conocido como "el muelle" que impide el oleaje reflectivo y aumenta el disipativo.

El sector La Remate fue estable durante toda la temporada por lo que el riesgo de pérdida de nidos por erosión fue igual durante toda la temporada. En cambio, en los sectores Cipara, La Peña y Varadero, el riesgo fue mayor en los primeros meses de la temporada (marzo-abril) y disminuyó a partir de mayo-junio.

Finalmente, a pesar que en Cipara la luz artificial es reciente y en San Juan, Unare y Querepare ésta tiene más de 10 años, la percepción que tienen la mayoría de los residentes costeros de la incidencia de la luz artificial sobre las tortugas marinas es la misma, es decir, que ésta no afecta a las tortugas marinas porque piensan que no les hace nada y de igual manera siguen saliendo a poner sus huevos o simplemente salen durante el día a desovar. Esto puede deberse a, según los pobladores, hace 30 años la Península de Paria, era importante para la anidación de la tortuga cabezona (Caretta caretta). El cardón era una especie poco común. Esta especie se comenzó a observar, hace 15 ó 20 años atrás, cuando el cabezón prácticamente había desaparecido en esta playa. En los últimos años ha aumentado la población de cardón y actualmente en Venezuela, Cipara y Querepare son las playas más importantes para su anidación. En el año de 1997, se registraron siete nidos de esta especie en Cipara y en 1998 cinco nidos (Guada 2000), en el año 2000 el número de nidos fue de 102.

\section{AGRADECIMIENTOS}

Al USFWS, al ICOMVIS y al BIODOC de La Universidad Nacional de Costa Rica. Al CICTMAR por su apoyo logístico, a la Estación de Investigaciones Marinas de Margarita de Fundación La Salle de Ciencias Naturales (EDIMAR) por su apoyo logístico y los análisis granulométricos. A Emilio Vargas, Jack Frazier y Hedelvy Guada.

\section{RESUMEN}

En Playa Cipara, Península de Paria, Venezuela, se evaluó el impacto de la luz artificial sobre la anidación de la tortuga cardón. Se estimó el número de anidaciones y su distribución espacial a lo largo de la playa entre los años sin y con iluminación artificial y entre segmentos de playa iluminada y oscura. Se hicieron entrevistas a los residentes para conocer su percepción sobre el impacto de 
la luz artificial hacia las tortugas marinas. Entre el 2000 y 2005 se registraron 1217 salidas de tortuga cardón; con 1056 nidos. El número de nidos con huevos dependió significativamente del año $(\mathrm{p}=0.035)$ al igual que el número de nidos totales $(\mathrm{p}=0.015)$. En los años previos a la electricidad (2000-2003) se observaron 743 salidas, de las cuales 661 con nido y 374 nidadas confirmadas. En los dos años (2004-2005) con iluminación artificial, se contaron 474 salidas con 395 nidos y 232 nidadas. La proporción de salidas con construcción de nido disminuyó significativamente $(p=0.005)$ en los años con luz eléctrica, pero no varió el éxito de desove $(\mathrm{p}=0.402)$. No se encontró diferencia significativa entre el número de salidas por metro de playa en los sectores oscuros y los iluminados $(\mathrm{p}=0.244)$, ni entre el número de nidos construidos $(\mathrm{p}=0.379)$, ni entre las anidaciones con desove $(\mathrm{p}=0.516)$. Tampoco en la proporción de las salidas totales que constituyeron anidaciones $(p=0.067)$ entre los sectores iluminados y oscuros, ni en la proporción de nidos exitosos ( $\mathrm{p}=0.833$ ). El volumen medio de arena por metro de playa fue mayor en La Peña, Cipara y La Remate y menor en Varadero $(\mathrm{p}<0.001)$, aumentando de abril a junio y posteriormente disminuyó un poco hasta agosto $(p<0.05)$. El porcentaje medio de gravas fue mayor en Varadero y La Peña y menor en La Remate y Cipara. La mayoría de los entrevistados consideraron que la luz artificial no afecta a las tortugas marinas.

Palabras clave: Dermochelys coriacea, playa Cipara, anidación, iluminación artificial, tortuga cardón, Venezuela, tortugas marinas.

\section{REFERENCIAS}

Bourgeois, S. 2007. Etude du succès reproductive de la tortue luth (Dermochelys coriacea) sur la plage de Pongara au Gabon: Devenir des nids et orientation des nouveau-nées. Thesis, Ecole Nationale Vétérinaire d'Alfort, Maisons-Alfort, Francia.

GETM-CSE/UICN 1995. Estrategia mundial para la conservación de las tortugas marinas. UICN/CSE Grupo especialista en Tortugas Marinas.

Carr, A. 1962. Orientation problems in the seas travel and terrestrial movements of marine turtles. Am. Sci. 50: 358-374.

Carr, A. \& L. Ogren. 1959. The ecology and migrations of sea turtles. 3. Dermochelys in Costa Rica. Am. Mus. Nov. 1958: 1-29.

Cea, M.A. 1998. Metodología cuantitativa. Estrategias y técnicas de investigación social. Síntesis S.A., Madrid, España.

Chacón, D. \& R. Arauz. 2001. Diagnóstico regional y planificación estratégica para la conservación de las tortugas marinas en Centroamérica. Red Regional para la Conservación de las Tortugas en Centroamérica. Fundación Acceso, San José, Costa Rica.

Cornelius, S.E. 1986. Sea turtles of Santa Rosa National Park. Fundación de Parques Nacionales, San José, Costa Rica.

Deem, S.L., F. Boussamba, A.Z. Nguema, G. Sounguet, S. Bourgeois, J. Cianciolo \& A. Formia. 2007. Artificial lights as a significant cause of morbidity of leatherback sea turtles in Pongara National Park, Gabon. Mar. Turt. News. 116: 15-17.

Eckert, K.L. 1987. Environmental unpredictability and leatherback sea turtle (Dermochelys coriacea) nest loss. Herpetologica 43: 315-323.

Eckert, K.L. \& S.A. Eckert. 1983. Tagging and nesting research of leatherback sea turtles (Dermochelys coriacea) on Sandy Point, St Croix, 1983. Ann. Rept. U.S. Fish Wildl. Ser. MIN 54-8480119.

Emery, K.O. 1961. A simple method of measuring beach profiles. Limnol. Ocean. 6: 90-93.

Flemming, E. 2001. Swimming against the tide: recent surveys of exploitation, trade, and management of marine turtles in the northern Caribbean. TRAFFIC, Washington D.C., EEUU.

Francia, A.G. 2004. Incidencia humana sobre la anidación de tortugas marinas: recolección de huevos y desarrollo urbano en playa Junquillal, Guanacaste, Costa Rica. Tesis de Maestría. PRMVS-UNA, Heredia, Costa Rica.

Guada, H.J. \& G. Solé. 2000. WIDECAST: Plan de acción para la recuperación de las tortugas marinas de Venezuela (A. Suárez, ed). Informe Técnico del PAC No. 39. UNEP Caribbean Environment Programme.

Guada, H.J., A. Fallabrino, A.C. Martínez, D.A. Muñoz, M.A. Rondón, S. Gómez, M. Morisson, L. Florez, A.M. Santana, G. Idrobo, J.L. Di Paola, E. Carabelli, L. Veiga, A. Naveda, D. Urbano \& C. Urbano. 2002. Proyecto de Investigación y Conservación de tortugas marinas en Cipara, Península de Paria, Estado Sucre, Venezuela: Resultados preliminares de la temporada de anidación del 2000. Noticiero de Tortugas Marinas 95: $17-18$.

Hernández, R. 2002. Evaluación de la anidación de la tortuga cardón, Dermochelys coriacea (Vandelli, 1761) (Reptilia: Dermochelyidae), en playa Parguito, Isla de Margarita, durante la temporada 2001. Tesis de Grado. Fundación La Salle. Instituto Universitario de Tecnología del Mar, Punta de Piedras, Venezuela. 
Kennett, R., N. Munungurritj \& D. Yunupingu. 2004. Migration patterns of marine turtles in the Gulf of Carpentaria, northern Australia: Implications for aboriginal management. Wil. Res. 31: 241-248.

Llano, M. 1987. Dinámica sedimentaria de la laguna de Punta de Piedras I: Época de mareas mínima. Mem. Soc. Ccs. Nat. La Salle 48: 37-76.

Lutcavage, M., P. Plotkin, B. Witherington \& P. Lutz. 1997. Human impacts on sea turtle survival. Capítulo 15. p. 387-410. In P.L. Lutz \& J.A. Musick (eds.). The Biology of Sea Turtles. CRC, Nueva York, EEUU.

Mann, T.M. 1977. Impact of developed coastline on nesting and hatchling sea turtles in Southeastern Florida. Master's Thesis, Florida Atlantic University, Boca Raton. EEUU.

Mattison, C., C. Burney \& L. Fisher. 1993. Trends in the spatial distribution of sea turtle activity on an urban beach (1981-1992). p. 102-104. In B. Schroeder \& B. Witherington (eds.). Proceedings of the Thirteenth Annual Symposium on Sea Turtle Biology and Conservation. NOAA Technical Memorandum NMFS-SEFC-341.

Mortimer, J.A. 1982. Factors affecting beach selection by nesting sea turtles. p. 45-51. In K.A. Bjorndal (ed.). Biology and Conservation of Sea Turtles. Smithsonian Institution, Washington, D.C, EEUU.

Mortimer, J.A. 1990. The influence of beach sand characteristics on the nesting behavior and clutch survival of green turtles (Chelonia mydas). Copeia 1990: 802817.

Mrosovsky, N. 1983. Ecology and nest-site selection of leatherback turtles, Dermochelys coriacea. Biol. Cons. 26: 47-56.

Mrosovsky, N. \& A. Carr. 1967. Preference for light of short wavelengths in hatchling green sea turtles, Chelonia mydas, tested on their natural nesting beaches. Behaviour 28: 217-231.

Mrosvsky, N. \& S.J. Shettleworth. 1975. On the orientation circle of the leatherback turtle, Dermochelys coriacea. Anim. Behav. 23: 195-208.

Murphy, T. 1985. Telemetric monitoring of nesting loggerhead sea turtles subjected to disturbance on the beach. Unpublished paper presented at the Fifth Annual Workshop on Sea Turtle Biology and Conservation, 13-16 February 1985.

Parra, L.I. 2002. Diagnóstico de la situación actual de las poblaciones de tortugas marinas en la costa occidental del Golfo de Venezuela, Estado Zulia. Trabajo Especial de Grado. LUZ, Maracaibo, Venezuela.

Raymond, P. 1984. Desorientación de neonatos de tortugas marinas y la iluminación artificial de las playas. Informe del Centro para la Educación Ambiental del Fondo para el Rescate de la Tortuga Marina.

Rondón, M. 2003. Informe del Proyecto de Investigación y Conservación de Tortugas Marinas. Cipara, Península de Paria. Temporada 2003. Informe Técnico de CICTMAR

Salmon, M., R. Reiners, C. Lavin \& J. Wyneken. 1995. Behavior of loggerhead sea turtles on an urban beach. I. Correlates of nest placement. J. Herp. 29: 560-567.

Shepard, F. 1954. Nomenclatura based on San-Silt-Clay ratios. J. Sed. Petr. 24: 151-158.

Schroeder, B. \& S. Murphy. 2000. Prospecciones poblacionales (terrestres y aéreas) en playas de anidación, p. 51-61. In K.L. Eckert, K.A. Bjorndal, F.A. AbreuGrobois \& M. Donnelly (eds.). 2000 (Traducido al español). Técnicas de investigación y manejo para la conservación de las tortugas marinas. UICN/CSE Grupo especialista en Tortugas Marinas Publicación $\mathrm{N}^{\circ} 4$.

Silman, R., I. Vargas \& S. Troëng. 2002. Tortugas marinas. Guía Educativa. CCC.

Sokal, R.R. \& F.J. Rohlf. 1981. Biometry. W. H. Freeman and Co., San Francisco, California, EEUU.

Spotila, J.R., R.D. Reina, A.C. Steyermark, P.T. Plotkin \& F.V. Paladino. 2000. Pacific leatherback turtles face extinction. Nature 405: 529-530.

Talbert, O.J Jr., S.E. Stancyk, J.M. Dean \& J.M. Will. 1980. Nesting activity of the loggerhead turtle (Caretta caretta) in South Carolina. I. A rookery in transition. Copeia 1980: 709-719.

Weishampel, J.F., D.A. Bagley, L.M. Ehrhart \& B.L. Rodenbeck. 2003. Spatiotemporal patterns of annual sea turtle nesting behaviors along an East Central Florida beach. Biol. Cons. 110: 295-303.

Williams-Walls, N., J.O’Hara, R.M. Gallagher, D.F. Worth, B.D. Peery \& J.R. Wilcox. 1983. Spatial and temporal trends of sea turtle nesting on Hutchinson Island, Florida, 1971-1979. Bull. Mar. Sci. 33: 55-66.

Witherington, B.E. 1992. Behavioral responses of nesting sea turtles to artificial lighting. Herpetologica 48: 31-39. 
Witherington, B.E. 2000. Reducción de las amenazas al hábitat de anidación, p. 201-210. In K.L. Eckert, K.A. Bjorndal, F.A. Abreu-Grobois \& M. Donnelly (eds.). 2000 (Traducido al español). Técnicas de investigación y manejo para la conservación de las tortugas marinas. UICN/CSE Grupo especialista en Tortugas Marinas Publicación $\mathrm{N}^{\circ} 4$

Witherington, B.E. \& K.A. Bjorndal. 1991. Influences of wavelength and intensity on hatchling sea turtle phototaxis: Implications for sea-finding behaviour. Copeia 1991: 1060-1069.

Witherington, B.E. \& R.E. Martin. 2003. Entendiendo, evaluando y solucionando los problemas de contaminación de luz en playas de anidación de tortugas marinas. Florida Marine Research Institute Technical Report TR-2- $75 \mathrm{p}$.

Wood, D.W. \& K.A. Bjorndal. 2000. Relation of temperature, moisture, salinity, and slope to nest selection in loggerhead sea turtles. Copeia 2000: 119-128.

Worth, D.F. \& J.B. Smith. 1976. Marine turtle nesting on Hutchinson Island, Florida, in 1973. Flor. Mar. Res. Publ. 18: 1-17.

\section{REFERENCIA DE INTERNET}

IUCN 2008. 2008 IUCN Red List of Threatened Species; www.iucnredlist.org (consultado 18 noviembre 2008). 